\title{
La formation et l'emploi des diplômes en beaux-arts, en humanités et en sciences sociales au Canada
}

\author{
AVIGDOR FARINE*, RON KNOWLES**
}

\begin{abstract}
RESUME
L'enseignement supérieur au Canada a connu une expansion considérable durant la dernière décennie. La population étudiante croissa de $15 \%$ par an, ce qui porta la proportion du groupe d'âge 18-24 ans au post-secondaire de 10.2\% en 1960-61 à $17.4 \%$ pour les hommes et de $3.3 \%$ à $9.8 \%$ pour les femmes. Parallèlement, les sommes affectées à ce niveau d'enseignement augmentèrent de $20 \%$ par an. Au Québec, par exemple, tandis que le P.N.B. augmentait de $8.6 \%$ par an et que les effectifs étudiants progressaient de $13 \%$, les dépenses de fonctionnement de l'enseignement supérieur ont crû de $23.5 \%$ par an. ${ }^{1}$

Le coût de cet enseignement et une situation économique difficile nous amènent à revoir la question de la formation et l'emploi. Dans cet article nous allons nous limiter aux diplomés en beaux-arts, en humanités et en sciences sociales au Canada, ayant traité ailleurs des autres grands groupes de la main-d'oeuvre hautement qualifiée $(M H Q)^{2}$ Les données de l'enquête post-censitaire sur la MHQ menée par Statistique Canada en 1973 pour le ministère d'Etat aux sciences et à la technologie servent de base à notre travail. ${ }^{3}$
\end{abstract}

\begin{abstract}
Higher education in Canada has experienced a large expansion in the last decade. The student population grew by 15 percent per year, which raised the proportion of the 18-24 year old age group participating in post-secondary education from 10.2 percent in 1960-1961 to17.4 percent for men and from 3.3 percent to 9.8 percent for women. During the same period, the support allocated to this sector of education increased by 20 percent per year. In Quebec, for example, while the P.N.B. increased by 8.6 per year and the number of students grew by 13 percent, the operating costs of higher education grew by 23.5 percent per year. ${ }^{1}$

The cost of this education and the difficult economic situation lead us to review the question of development and role. In this article, we shall limit ourselves to degrees in fine arts, humanities, and social sciences in Canada, having dealt elsewhere with the other major
\end{abstract}

*Université de Montréal

**Ministère d'Etat aux Sciences et à la Technologie 
categories of highly qualified manpower $(M H Q)^{2}$. The post-census inquiry data on $M H Q$ prepared in 1973 by Statistics Canada for the Ministry of State for science and technology serves as the basis for our study. ${ }^{3}$

\section{Revue de quelques écrits sur le sujet}

Durant la dernière décennie, une société en évolution, des besoins urgents en main-d'oeuvre qualifiée et les attentes créées par le prolongement des études ont poussé des milliers d'étudiants vers les collèges et les universités. Au Québec, par exemple, des panneaux sur les routes proclamant "qui s'instruit s'enrichit" montraient le désir d'atteindre par des études supérieures une meilleure situation économique et une mobilité sociale qui l'accompagne. La poursuite des cours universitaires a été aussi encouragée par des études sur la rentabilité de la formation universitaire "démontrant" qu'il est valable de prolonger la scolarité, suite au meilleur taux de rendement qu'un grade universitaire plus élevé rapportait à son détenteur, par comparaison au détenteur d'un grade moins élevé.

La difficulté des diplômés en arts et sciences à trouver un emploi a été ressentie à la fin de 1969. Jusqu'alors, la demande de diplômés dépassa l'offre. La possession d'un grade universitaire était une garantie d'obtention d'un emploi correspondant au domaine d'études accomplies. Les difficultés actuelles d'embauche sont dues à la saturation du marché, à la concurrence d'un plus grand nombre de diplômés pour les mêmes postes et à l'augmentation de spécialisation exigée pour la plupart des emplois. Des grades supérieurs semblent être requis pour remplir des fonctions qui n'exigeaient qu'un premier grade universitaire. ${ }^{4}$

Par ailleurs, l'optimisme des années 1960 vis-à-vis la planification de l'éducation et la concordance entre la formation et l'emploi n'a plus cours aujourd'hui. Dodge, par exemple, constate que les revenus élevés des diplômés résultent davantage du "barrage" que constitue le diplôme, plutôt que des effets de la formation universitaire sur la productivité du diplômé. ${ }^{5}$ Il soutient qu'il existe une exagération des taux estimés des études supérieures et de la rentabilité de l'investissement dans ces études. En outre, une étude récente préparée pour le Conseil Economique du Canada trouve que les bénéfices d'une scolarité prolongée ne sont plus évidents par eux-mêmes. ${ }^{6}$ L'étude conclut que la confiance du public vis-à-vis le système scolaire va probablement fléchir, suite à la critique grandissante à l'égard du taux de rendement de l'investissement en éducation. Dans son examen des perspectives de la formation universitaire, Harvey dit que, selon toute apparence, un sous-emploi cause un déclin du taux de rendement individuel et social. ${ }^{7}$ Une surqualification du travailleur mène aussi à des attentes de travail non remplies, donc à une insatisfaction au travail.

\section{La MHQ et la relation formation/emploi}

Dans les pays économiquement développés, les politiques autonomes de main-d'oeuvre n'ont été instaurées que vers les années 1945. Dans les années 1970, les problèmes d'emploi ne sont plus seulement ceux du personnel ouvrier, mais aussi ceux du personnel hautement qualifié qui représente numeriquement et politiquement une puissance croissante. ${ }^{8} \mathrm{Au}$ Canada, ce personnel hautement qualifié provient presqu'en totalité des institutions d'enseignement post-secondaire. 
La formation universitaire offre aux individus les moyens d'acquérir les compétences nécessaires à l'exécution d'une profession. On s'attend à ce que les programmes de formation soient en relation étroite avec le marché du travail, surtout avec les emplois qu'occuperont les diplômés au début de leur carrière professionnelle. Toutefois, cette relation peut s'avérer moins étroite par la suite, à cause d'une mobilité de travail continuelle. Par ailleurs, les modifications technologiques rapides liées à la transformation des rapports entre l'offre et la demande de diplômés, et la mobilité interprofessionnelle de ces derniers, sont des sources de difficultés rencontrées dans la recherche de correspondance entre la formation et l'emploi.

Ainsi, la complexité des liens entre la formation et l'emploi ne permet pas de trouver des réponses immédiates et directes aux différentes questions soulevées. Car, il n'existe pas de moyens satisfaisants qui permettent de spécifier les besoins éducatifs exigés par la majorité des professions. L'O.C.D.E. note les problèmes de classification de la main-d'oeuvre et, faute de connaissances, ne suggère que la conversion des besoins de chaque catégorie d'emploi en besoins classés par niveau d'éducation. ${ }^{9}$ Dans ce contexte, pouvons-nous dire qu'en établissant leurs programmes, les universités devraient tenir compte des besoins du marché. De leur côté, les étudiants choisissent de suivre une formation en fonction des compétences qui semblent être nécessaires ou utiles pour la carrière qu'ils désirent poursuivre. Malheureusement, la traduction des fonctions professionnelles en exigences d'études ou de types de formation appropriées se font généralement sur des suppositions arbitraires. ${ }^{10}$

\section{Prévision des besoins en main-d'oeuvre et choix du domaine d'études}

Holland et al. soulignent la multitude des variables à considérer et les contraintes existantes dans la prévision de la MHQ. ${ }^{11}$ L'analyse de ces variables requiert la connaissance du marché et des facteurs qui influencent la prise de décision des employeurs, des employés, des associations professionnelles, des étudiants et de ceux qui décident des politiques en matière d'éducation. Quant aux étudiants, des contraintes psychologiques et sociales conditionnent souvent leurs aptitudes, leurs habiletés et leurs intérêts. Ces facteurs sont liés au choix du domaine d'études et conduisent l'étudiant à choisir parfois une discipline rapportant moins de rendement monétaire, en art, par exemple, plutôt qu'en génie. Selon ces auteurs, les contraintes financières ne semblent pas avoir une influence principale sur les décisions éducatives des étudiants. En tout cas, une meilleure information sur les emplois peut devenir un facteur important dans leur prise de décision.

Dans une étude sur les facteurs qui influencent le choix de carrière des étudiants, Dodge et al. trouvent qu'un tiers de leurs répondants ont préféré des carrières d'un moindre rendement monétaire. ${ }^{12}$ "L'irrationnel" du choix d'une carrière est dû, en partie, à la difficulté de mesurer les valeurs non-pécuniaires de cette carrière. Les auteurs se sont interrogés sur les aspects du modèle néo-classique de choix de carrière: l'actualisation des gains à travers le temps et l'information à l'égard des carrières. Ils rapportent que seulement $50 \%$ des répondants actualisent leurs gains futurs à un taux positif et que 35\% d'eux arrivent même à des taux implicitement négatifs. Les auteurs concluent qu'en général, les étudiants manquent d'information à l'égard des salaires; ils sous-estiment les salaires initiaux pour la majorité des emplois, mais surestiment le revenu à travers le temps de la carrière de leur choix.

Nous terminons par les remarques de la Carnegie Commission on Higher Education. Une 
nouvelle période se dessine, dit-elle, où la question de débouchés devient un problème. Plus de diplômés se feront concurrence pour les mêmes postes. Environ 30\% des diplômés des collèges aux Etats-Unis travaillent comme cols-bleus, vendeurs ou employés de bureau. Un sous-emploi de la formation reçue en résulte. Les diplômés devront s'adapter à cette nouvelle situation. ${ }^{13}$

\section{Analyse des tableaux}

Quel est le principal domaine d'études selon le dernier diplôme le plus élevé détenu par les répondants? Quelle profession exercent-ils? Quel est le degréde mobilité interprofessionnelle? Est-ce que historien, géographe, sociologue, et on peut continuer la liste), sont des professions? Nous allons essayer de répondre à ces questions à l'aide des tableaux en Appendice et nous terminerons par des commentaires résultant des données.

En 1973, Statistique Canada a mené une enquête auprès de 138,000 individus qui ont déclaré lors du recensement de 1971 détenir un diplôme universitaire. Les résultats de cette enquête viennent d'être complétés et les tableaux que nous avons établis présentent des aspects d'études et de travail que nous désirons examiner.

Deux remarques s'imposent. Les données de Statistique Canada ont été recueillies selon "le dernier diplôme le plus élevé obtenu". Cette décision est justifiée du fait que la classification par dernier diplôme correspond à l'expérience de travail présente ou récente des diplômés. Néanmoins, cette classification a un point faible. Par exemple, un individu qui a son premier diplôme en droit et son dernier en administration hospitalière n'est pas classifié comme avocat mais comme administrateur d'hôpital. Du point de vue de la quantification de la MHQ, la valeur empirique de son diplôme en droit n'apparait pas.

Dans les tableaux de Statistique Canada, chaque domaine d'études comportait une liste détaillée des groupes de professions (par exemple, Education: sans profession, profession 1, profession 2, etc.; Beaux-arts: sans profession, profession 1, profession 2,). Nous avons eu à les condenser. Bien que ce regroupement fait perdre la spécificité des professions, il permet de voir la MHQ par grands groupes.

\section{Croisement du domaine d'études et de l'emploi (tableaux 1 et 2 )}

Ces tableaux du principal domaine d'études croisés à l'emploi le plus longtemps occupé au cours des 12 derniers mois reflètent l'image de la mobilité interprofessionnelle et de la relation formation/emploi. Cette dernière ne semble pas être étroite dans la grande majorité des disciplines. Elle semble l'être en droit, en théologie, en administration hospitalière, en comptabilité et en service social. Elle est presque nulle dans les humanités et certaines disciplines en sciences sociales. Par exemple, seulement $5.8 \%$ des sociologues, $10 \%$ des diplômés en beaux-arts, $12.5 \%$ des économistes et $32.7 \%$ des psychologues cliniciens exercent directement leur propre profession. Où travaillent les autres? Ils occupent des emplois en enseignement, dans les humanités et en sciences sociales; ils sont dans l'administration et la gestion des entreprises; ils sont occupés dans des activités connexes (cols-blancs) ou oeuvrent dans des activités étrangères à leur formation. Environ $18 \%$ des individus provenant des beauxarts et des humanités et $10 \%$ de ceux des sciences sociales sont en dehors de de la maind'oeuvre active. 
Beaux-arts et humanités correspondance entre la formation et l'emploi

\begin{tabular}{|c|c|c|c|c|c|c|c|c|c|}
\hline \multirow{2}{*}{ Principal domaine d'études } & \multirow{2}{*}{$\begin{array}{c}\text { Total } \\
\text { diplômés }\end{array}$} & \multicolumn{7}{|c|}{ Emploi } & \multirow{2}{*}{$\begin{array}{l}\text { Total \% } \\
\text { emploi** }\end{array}$} \\
\hline & & $1^{*}$ & 2 & 3 & 4 & 5 & 6 & 7 & \\
\hline Arts (y compris peinture, gravure et sculpture) & 4,595 & 10.7 & 24.6 & 10.0 & & & 10.0 & 17.3 & 72.3 \\
\hline Musique & 4,340 & 9.3 & 46.3 & & & & & 22.8 & 78.4 \\
\hline Etudes et langues classiques & 2,805 & & 42.2 & 3.2 & 11.4 & & & 36.2 & 93.0 \\
\hline Langue et littérature française & 9,705 & & 51.7 & 4.8 & 7.3 & & & 15.3 & 79.1 \\
\hline Langue et littérature anglaise & 24,780 & 3.1 & 39.8 & 6.6 & 11.0 & & & 24.3 & 84.8 \\
\hline Langue et littératures modernes & 5,030 & & 35.4 & 6.2 & 9.0 & & & 21.1 & 71.7 \\
\hline Création littéraire (écrivains, journalistes, éditeurs) & 1,805 & 23.8 & 14.4 & & 21.3 & & & 13.3 & 72.8 \\
\hline Histoire & 20,140 & & 39.7 & & 14.5 & 11.5 & & 24.9 & 90.6 \\
\hline Philosophie & 6,830 & & 33.0 & & 14.9 & 9.3 & & 13.8 & 71.0 \\
\hline Théologie et sc. religieuses & 15,985 & 46.9 & 16.8 & & 7.8 & 56.3 & & 6.3 & 87.2 \\
\hline Traduction & 320 & 58.3 & & & 14.0 & & 26.6 & 3.1 & 90.6 \\
\hline Bibliothéconomie et classement & 7,045 & & 3.7 & & & & 9.2 & 17.7 & 88.9 \\
\hline GRAND TOTAL & 103,380 & & & & & & & & \\
\hline
\end{tabular}

*1. dans sa profession; 2. en enseignement; 3. autre profession en beaux-arts et humanités; 4. administration, gestion et connexe; 5. clergé; 6. autres (y compris les non-spécialisés); 7. ne travaille pas.

** Le total de l'emploi est au-dessous de 100\%, n'ayant considéré que les groupes majeurs de professions. 
Tableau 2

Sciences sociales

correspondance entre la formation et l'emploi

\begin{tabular}{|c|c|c|c|c|c|c|c|c|}
\hline \multirow{2}{*}{ Principal domaine d'études } & \multirow{2}{*}{$\begin{array}{l}\text { Total } \\
\text { diplômés }\end{array}$} & \multicolumn{6}{|c|}{ Emploi } & \multirow{2}{*}{$\begin{array}{l}\text { Total\% } \\
\text { emploi** }\end{array}$} \\
\hline & & $1 *$ & 2 & 3 & 4 & 5 & 6 & \\
\hline Anthropologie & 1,750 & 5.7 & 40.8 & 5.1 & 9.2 & 10.0 & 5.0 & 75.8 \\
\hline Archéologie & 180 & 33.3 & 19.5 & 13.9 & 13.9 & & & 80.6 \\
\hline Etudes canadiennes, etc. & 430 & & 33.7 & 10.5 & 2.3 & & 25.6 & 72.1 \\
\hline Administration et gestion d'établissements & 545 & 13.8 & 4.6 & 50.5 & 1.8 & 7.3 & 7.3 & 85.3 \\
\hline Administration publique & 885 & & 4.5 & 48.0 & 7.3 & 7.3 & 5.6 & 72.7 \\
\hline Administration hospitalière & 435 & 44.8 & & 21.5 & 8.1 & & & 74.4 \\
\hline Commerce, finances, rel. ind. & 35,015 & & 4.7 & 55.3 & 4.5 & 3.2 & 8.3 & 76.0 \\
\hline Secrétariat & 835 & 14.4 & 15.6 & 9.6 & & 14.4 & 10.0 & 64.0 \\
\hline Sciences économiques & 15,955 & 12.5 & 10.1 & 32.7 & 14.4 & 8.0 & 7.9 & 85.6 \\
\hline Economie agricole & 1,385 & 11.9 & & 30.1 & & 10.5 & 5.1 & 57.6 \\
\hline
\end{tabular}


Jusqu'en 1973, l'enseignement continue d'être le premier secteur d'activité des diplômés (33.5\% en moyenne). Il l'est de beaucoup plus pour les diplômés en musique, en lettres, en histoire ou en géographie. Ceci correspond à une étude de l'O.C.D.E. où, selon le recensement de 1960-61 dans différents pays membres, les personnes employées par les services d'enseignement représentent entre un cinquième et un tiers de l'ensemble de la MHQ. ${ }^{14}$ Dans les professions où la formation est déterminée par certification, telles que le droit, la théologie ou le service social, la relation formation/emploi est plus étroite. Il est possible que la certification serve de sélection et de "barrage" à l'entrée de la profession, rendant ainsi la relation plus étroite.

Quant aux diplômés en traduction, bien que $46.9 \%$ exercent leur métier, $26.6 \%$ remplissent des fonctions étrangères à leur formation et travaillent comme non-spécialisés. De même, 9.2\% des diplômés err bibliothéconomie et classement (archivistes) indiquent remplir des fonctions de non-spécialisés. Les psychologues ne semblent pas exercer en grand nombre $(8.8 \%)$ leur propre profession. Notons que les données incluent également les détenteurs d'un baccalauréat en psychologie qui ne sont pas considérés en tant que psychologues par la Corporation des psychologues. Car pour pratiquer, il faut être membre de cette corporation et posséder au moins la maítrise en psychologie.

\section{L'offre et la demande de diplômés (tableau 3)}

Ce tableau apporte plus de clarté en présentant les professions par grands groupes. L'offre signifie la proportion de diplômés d'une discipline qui exercent la profession correspondant à cette discipline. La demande signifie la proportion d'individus qui exercent une profession et qui détiennent aussi leur dernier diplôme dans la discipline respective à leur profession. Ainsi, du point de vue de l'offre et par ordre d'importance croissant, les diplômés en beauxarts pratiquent les beaux-arts à $12 \%$, ceux en sciences humaines exercent leur profession à $19 \%$ et ceux en sciences sociales (le droit inclus) le font à $54 \%$.

Inversement, du point de vue de la demande de diplômés, $68 \%$ des professionnels en sciences humaines, $52 \%$ de ceux en sciences sociales, mais seulement $18 \%$ de ceux en beauxarts détiennent aussi leur dernier diplôme dans la discipline respective à leur profession.

La différence entre l'offre et la demande de diplômés est grande dans les humanités. Ces individus ont des diplômes universitaires (l'offre), mais ne travaillent pas en grand nombre dans le domaine de leur spécialisation (la demande). La même situation est constatée chez les diplômés en beaux-arts et certaines disciplines en sciences sociales.

Le tableau 3 permet la comparaison avec d'autres disciplines. Du point de vue de l'offre, les sciences agricoles et la biologie, les mathématiques et les sciences physiques souffrent aussi d'un manque de relation étroite entre la formation et l'emploi. Nous avons auparavant posé la question, est-ce que historien (ou autre) est une profession? On peut se le demander aussi à l'égard de mathématicien.

\section{Sommaire}

Cet article a souligné les difficultés rencontrées dans la recherche de correspondance entre la formation et l'emploi en beaux-arts, en humanités et en sciences sociales. De nombreux 
19 La formation et l'emploi des diplômes en beaux-arts, en humanités et en sciences sociales au Canada

Tableau 3

L'offre et la demande de diplomes par grands groupes

a) L'offre

$\begin{array}{lll}\text { Principal domaine d'etudes } & \begin{array}{l}\text { Pourcentage d'individus } \\ \text { exerçant des professions } \\ \text { correspondantes }\end{array} & \begin{array}{l}\text { La disponibilité } \\ \text { relative de la }\end{array} \\ & \text { main-d'oeuvre }\end{array}$

Beaux-arts

12.0

$1 *$

Sc. agricoles et biologiques

13.0

2

Sciences humaines

19.0

3

Mathématiques

21.0

Sciences physiques

29.0

4

Génie et architecture

51.0

5

Sciences sociales

54.0

6

Education

62.0

7

Santé

77.0

8

9

*Par ordre d'importance

b) La demande

\begin{tabular}{lll} 
Profession & $\begin{array}{l}\text { Pourcentage d'individus } \\
\text { détenant leur dernier }\end{array}$ & $\begin{array}{l}\text { La correspondance } \\
\text { entre la formation } \\
\text { diplôme dans la discipline } \\
\text { correspondante }\end{array}$ \\
\hline
\end{tabular}

Santé

$91.0 \quad 9 *$

Génie et architecture

89.0

$9^{*}$

Agriculture et biologie

85.0

8

Sciences humaines

68.0

7

Sciences sociales

52.0

6

Sciences physiques

43.0

43.0

40.0

Mathématiques

18.0

5

4

3

Beaux-arts

2

1

*9- la plus étroite, 1- la moins étroite.

facteurs contribuent au fait qu'un grand nombre d'individus exercent des fonctions étrangères à leur dernière formation. 
Les tableaux ont mis en relief la mobilité interprofessionnelle qui prend de l'envergure dans une période de 5 à 10 ans. Cette mobilité devrait être prise en considération dans la prévision de la MHQ. Cependant, cette prévision devrait plutôt s'appliquer aux domaines de la santé, du génie ou autres secteurs de la MHQ qui accusent une relation formation/ emploi plus étroite que les humanités et les sciences sociales.

Les données soulèvent quelques réflexions. Outre la transférabilité des habiletés d'une fonction à une autre dans beaucoup de professions, on perçoit que différentes formations permettent l'accès à une pluralité de proffessions et qu'une même profession peut être exercée par des individus ayant des formations différentes. Ceci souligne l'existence de complémentarité et de substitution dans tout capital humain. La formation sur le tas, formelle ou informelle, y contribue grandement.

L'enseignement a servi de premier secteur d'activité des diplômés. Cependant, la saturation des emplois en éducation posera plus de difficulté à atteindre un poste aux diplômés de certaines disciplines. Il semble nécessaire de créer un meilleur système d'information qui guiderait les nouveaux étudiants dans leur choix de discipline universitaire. D'après l'étude de Dodge et al., citée auparavant, les étudiants du premier cycle manquent d'information sur l'emploi; ils sont moins influencés par des aspects économiques futurs et leurs valeurs suivent plutôt les tendances de leur groupe d'âge. En effet, des facteurs biologiques et culturels (aptitudes et intérêts), l'orientation des études précédentes, les notes reçues au collège et d'autres facteurs interviennent dans le choix de cours. ${ }^{15}$

Plusieurs économistes, sociologues, psychologues, géographes et autres diplômés ne travaillent pas dans le domaine de leur dernière formation. Il semble que l'entrainement dans ces disciplines a pour but de développer certaines habiletés, sans relation étroite avec l'emploi. Il ressort qu'être historien, économiste ou autre qualifient un individu qui a développé des habiletés dans sa discipline qui peuvent être offertes comme professions spécifiques, mais ces habiletés sont transférables à une pluralité de professions.

Au terme de cette brève analyse, nous sommes loin de prétendre que la formation universitaire devrait être uniquement en fonction du marché du travail. Nous sommes conscients qu'une société qui tient à développer ses ressources humaines insiste aussi sur leur développement culturel. Nous nous éloignons également de la notion que l'effort universitaire au Canada, comme ailleurs, a répondu plus à la demande sociale et à l'aspect consommation plutôt qu'au besoin de la formation de la MHQ requise pour le développement économique. Mais une chose est certaine: tout diplôme a sa valeur économique, qu'il couronne des études classiques, générales ou techniques. Car il permet à son détenteur de trouver du travail, avec un degré de facilité ou de difficulté qui varie selon les études accomplies et la conjoncture économique. La difficulté des diplômés de certaines disciplines à trouver un emploi vérifie notre dernière constatation. 
2. La formation et l'emploi des diplômes en beaux-arts, en humanités et en sciences sociales au Canada

\section{Références}

1. Ministère de l'éducation, D.G.E.S., Statistiques de l'enseignement supérieur. Evolution de l'enseignement supérieur au Québec. 1961-1971. Québec, 1972, pp. 32-34.

2. A. Farine, "La mobilité in terprofessionnelle au Canada," Communication présentée au congrès annuel de l'Association Francophone d'Education Comparée, Paris, mai 1976.

3. Ministère d'état aux sciences et à la technologie, Enquête post-censitaire sur la main-d'oeuvre hautement qualifiée, 1973. Ottawa, avril 1974.

4. Department of Manpower and Immigration, Supply and Demand 1970: New University Graduates. Ottawa: The Queen's Printer, 1970, p. 11.

5. D. A. Dodge, "Occupational Wage Differentials, Occupational Licensing, and Returns to Investment in Education: An Exploratory Analysis," in S. Ostry ed., Canadian Higher Education in the Seventies. Ottawa: Conseil Economique du Canada, 1972), pp. 133-76.

6. J. B. Lacombe, "Some Economic Aspects of Education in Canada," Document de travail no 4, Conseil Economique du Canada. Ottawa, décembre 1974 (non-diffusé).

7. E. B. Harvey, "Canadian Higher Education and the Seventies," Interchange, Vol. 5, no 2 (1974), pp. 42-52.

8. O.C.D.E., L'utilisation du personnel hautement qualifié. Paris: O.C.D.E., 1973, p. 147.

9. O.C.D.E., Planification de l'enseignement. Problèmes d'organisation. Paris: O.C.D.E., 1966, p. 17.

10. R. Hollister, Evaluation technique de la première phase du Projet Régional Méditerrannéen. Paris: O.C.D.E., 1967, pp. 38, 93-95.

11. J. Holland et al. Manpower Forecasting and Educational Policy. A Report Prepared for the Commission on Post-Secondary Education in Ontario. Toronto: The Queen's Printer, 1971, pp. X, 56, 111, 133 et 157 .

12. D. A. Dodge et al., "Factors Influencing Career Choice of Students," Communication présentée au congrès annuel de l'Association Canadienne d'Economique, Saint-Jean, Terre-Neuve, juin 1974.

13. The Carnegie Commission on Higher Education, College Graduates and Jobs. New-York: McGraw-Hill, 1973, pp. 3-18.

14. O.C.D.E., Formation, recrutement et utilisation dans l'enseignement primaire et secondaire. Paris: O.C.D.E., 1971, p. 260.

15. A. Farine, avec la collaboration de P.-P. Proulx, Les diplômés de l'Université de Montréal sur le marché du travail. Université de Montréal, Centre de recherches en développement économique, Dossier 5, juin 1974 , p. 47. 